\title{
BMJ Open Study protocol of the Health4Life initiative: a cluster randomised controlled trial of an eHealth school- based program targeting multiple lifestyle risk behaviours among young Australians
}

\author{
Maree Teesson (D) , Katrina E Champion (D) ," Nicola C Newton (D) ,1 \\ Frances Kay-Lambkin (D) ,' Cath Chapman (D) ,' Louise Thornton (D) ,1 \\ Tim Slade (D) , ${ }^{1}$ Matthew Sunderland (D) , ${ }^{1}$ Katherine Mills, ${ }^{1}$ Lauren A Gardner (D) , \\ Belinda Parmenter (D) , ${ }^{3}$ David R Lubans (D) , ${ }^{4}$ Leanne Hides (i) ${ }^{5}$ \\ Nyanda McBride (D) , ${ }^{6}$ Steve Allsop, ${ }^{6}$ Bonnie J Spring (D) , ${ }^{7}$ Scarlett Smout, ${ }^{1}$ \\ Bridie Osman, ${ }^{1}$ The Health4Life Team
}

To cite: Teesson M, Champion KE, Newton NC, et al. Study protocol of the Health4Life initiative: a cluster randomised controlled trial of an eHealth school-based program targeting multiple lifestyle risk behaviours among young Australians. BMJ Open 2020;10:e035662. doi:10.1136/ bmjopen-2019-035662

- Prepublication history and additional material for this paper are available online. To view these files, please visit the journal online (http://dx.doi. org/10.1136/bmjopen-2019035662).

MT and KEC contributed equally.

Received 11 November 2019

Revised 11 May 2020

Accepted 29 May 2020

\section{Check for updates}

(c) Author(s) (or their employer(s)) 2020. Re-use permitted under CC BY-NC. No commercial re-use. See rights and permissions. Published by BMJ.

For numbered affiliations see end of article.

\section{Correspondence to} Dr Katrina E Champion; katrina.champion@sydney. edu.au

\section{ABSTRACT}

Introduction Lifestyle risk behaviours, including alcohol use, smoking, poor diet, physical inactivity, poor sleep (duration and/or quality) and sedentary recreational screen time ('the Big 6'), are strong determinants of chronic disease. These behaviours often emerge during adolescence and co-occur. School-based interventions have the potential to address risk factors prior to the onset of disease, yet few eHealth school-based interventions target multiple behaviours concurrently. This paper describes the protocol of the Health4Life Initiative, an eHealth school-based intervention that concurrently addresses the Big 6 risk behaviours among secondary school students.

Methods and analysis A multisite cluster randomised controlled trial will be conducted among year 7 students (11-13years old) from 72 Australian schools. Stratified block randomisation will be used to assign schools to either the Health4Life intervention or an active control (health education as usual). Health4Life consists of (1) six web-based cartoon modules and accompanying activities delivered during health education (once per week for 6 weeks), and a smartphone application (universal prevention), and (2) additional app content, for students engaging in two or more risk behaviours when they are in years 8 and 9 (selective prevention). Students will complete online self-report questionnaires at baseline, post intervention, and 12, 24 and 36 months after baseline Primary outcomes are consumption of sugar-sweetened beverages, moderate-to-vigorous physical activity, sleep duration, sedentary recreational screen time and uptake of alcohol and tobacco use.

Ethics and dissemination This study has been approved by the University of Sydney (2018/882), NSW Department of Education (SERAP no. 2019006), University of Queensland (2019000037), Curtin University (HRE2019-

\section{Strengths and limitations of this study}

- This study is a large, multisite randomised controlled trial of an eHealth intervention to prevent chronic disease risk among adolescents.

- The study will be implemented in 72 secondary schools (approximately 7200 students) across Australia, with participants completing assessments over 4 years.

- It is the first eHealth programme to concurrently target the 'Big 6' risk factors across the spectrum of universal and selective prevention.

Due to scale of the study, anthropometric and objective measurement were not feasible.

0083) and relevant Catholic school committees. Results will be presented to schools and findings disseminated via peer-reviewed journals and scientific conferences. This will be the first evaluation of an eHealth intervention, spanning both universal and selective prevention, to simultaneously target six key lifestyle risk factors among adolescents. Trial registration number Australian New Zealand Clinical Trials Registry (ACTRN12619000431123), 18 March 2019.

\section{INTRODUCTION}

Lifestyle risk behaviours, including alcohol use, smoking, poor diet, physical inactivity, poor sleep (long or short duration and/ or poor quality) and sedentary recreational screen time (the 'Big 6'), are among the strongest determinants of chronic disease, the leading cause of death worldwide. ${ }^{1}$ These risk behaviours typically emerge in adolescence 
and track into adulthood. ${ }^{2}$ Among Australian adolescents (aged 12-17 years), 66\% have consumed alcohol, $18 \%$ have smoked tobacco, ${ }^{3} 82 \%$ do not meet physical activity guidelines, ${ }^{4} 86 \%$ do not meet screen time guidelines, ${ }^{5} 52 \%$ of $15-16$ years old do not meet minimum sleep guidelines ${ }^{6}$ and $61 \%$ of 14-18years old consume sugar-sweetened beverages (SSBs) in a single day. ${ }^{7}$ These risk behaviours are associated with poor health outcomes in adolescence, such as obesity ${ }^{8}$ and mental health problems, ${ }^{9}$ and collectively, are associated with an increased risk of cardiovascular disease, type 2 diabetes, mental disorders and all-cause mortality in adults. ${ }^{1011}$

Not only is each of these risk factors prevalent on its own, but they tend to co-occur. ${ }^{12}$ For example, young people who use alcohol and other drugs are also more likely to eat poorly and be sedentary. ${ }^{13}$ Importantly, engaging in multiple risk behaviours increases the risk of chronic disease and mortality, more so than the additive effects of single behaviours, ${ }^{14-16}$ and has been associated with higher rates of mental health problems. ${ }^{17}$ In recognition of this, interventions targeting changes in multiple health behaviours concurrently ${ }^{18}$ are now being designed. This approach is grounded in 'transfer theory', ${ }^{19}$ in which new skills and knowledge that facilitate change in one behaviour are transferred to another context, ${ }^{20}$ resulting in a 'tag-along' healthy change in other behaviours without additional effort. ${ }^{21} 22$ In addition, previous research suggests that multiple health behaviour change interventions for adolescents are potentially more efficient $^{23}$ and cost effective ${ }^{24}$ than interventions targeting single behaviours. ${ }^{25} 26$

Schools are ideal settings to implement healthy lifestyle interventions as they provide access to large numbers of students at a critical time period, are the place where students spend most of their waking hours, and, in many countries, including Australia, have a mandated health education curriculum. Further, they have been shown to be effective settings for the promotion of multiple health behaviour change. ${ }^{27}$ eHealth interventions (ie, delivered via the internet, computers, tablets, mobile technology, or tele-health) delivered at school may offer a number of potential advantages, including increased engagement, fidelity and scalability. ${ }^{28}$ When looking specifically at eHealth school-based programmes targeting multiple lifestyle risk behaviours, a recent meta-analysis ${ }^{29}$ found such interventions to be effective in relation to diet, physical activity and screen time; however, effects were small and short-lived and studies were generally of low quality. Further, there were no existing eHealth school-based interventions that targeted all of the Big 6. Given these findings, randomised controlled trials (RCTs) using large, representative samples to test the effectiveness of eHealth school-based programmes targeting multiple lifestyle risk behaviours are needed.

To address these gaps, we developed the Health4Life Initiative, an eHealth intervention that aims to empower adolescents to reduce chronic disease risk, and improve current physical and mental health, by simultaneously targeting the Big 6 risk factors. This study aims to evaluate the effectiveness of Health4Life among adolescents through a multisite cluster RCT. It is hypothesised that Health4Life will be more effective than an active control group (health education as usual) in:

1. Delaying uptake of alcohol use.

2. Delaying uptake of tobacco use.

3. Reducing sedentary recreational screen time.

4. Reducing the decline in moderate-to-vigorous physical activity (MVPA).

5. Reducing consumption of SSBs.

6. Increasing sleep (among those with short duration) and decreasing sleep (among those with long duration).

\section{METHODS AND ANALYSIS}

\section{Study design}

A cluster RCT will be conducted among year 7 students at 72 independent, catholic and government secondary schools in New South Wales (NSW), Western Australia (WA) and Queensland (QLD), Australia, from 2019 to 2022. Due to differences in the number of available schools across states, half of the sample will be recruited from NSW (25\% from Greater Sydney and 25\% from other regions), while $25 \%$ will be recruited each from WA and QLD. Cluster randomisation will be used to avoid contamination of the control group with the intervention group through student and staff communication. Schools will be randomly allocated to either the Health4Life intervention or to an active control group (health education as usual). The protocol was written in accordance with the Standard Protocol Items: Recommendations for Interventional Trials statement.

\section{Sample size calculations}

The minimal detectable effect size associated with the health behaviours at the final time point (ie, 36 months) was estimated using power calculations developed by Heo and Leon $^{30}$ for linear mixed regression models taking into account the longitudinal and clustered nature of the data. The alpha level was set at a conservative 0.008 level based on a Bonferroni correction $(\mathrm{a}=0.05 / 6)$ to account for testing of multiple outcomes. Power was set at 0.8 ; the correlation among level 1 data (repeated measurements over time within student) was set at 0.60 and the number of time points set at 5 . The formula described by Heo and Leon ${ }^{30}$ does not require estimates of intraclass correlation coefficients at the student (level 2) or school (level 3) level. Analysis will be undertaken within each state separately and thus the trial will be powered to detect an effect size in the states with the smallest recruitment numbers. Data will also be pooled across states and analysed. With an anticipated student retention rate of $70 \%$ in each school, we plan to recruit 100 students from 72 schools (36 schools in NSW, with 18 from Greater Sydney and 18 from other regions, for example, Hunter and Illawarra, and 18 each in WA and QLD) to achieve 
sufficient power to detect an effect size of 0.158 . An effect size of 0.158 is based on the targeted behaviour with the lowest anticipated prevalence at baseline, that is, alcohol use. An effect size of 0.158 is associated with an OR of 0.75 and is a conservative estimate compared with previous school-based trials of universal substance use prevention programmes. ${ }^{31}$ Economic modelling indicates that even small effects for alcohol education programmes can have substantial benefits on a population level. ${ }^{32}$

\section{Inclusion and exclusion criteria}

Eligible participants will be year 7 students (aged approximately 11-13 years) attending participating schools who are fluent in English. Students will be required to provide active informed consent and only students with parental consent are eligible to participate. Schools will be required to be based in NSW, WA or QLD and have a minimum of 30 year 7 students enrolled in 2019.

\section{Procedure}

Recruitment of schools

The 72 schools will be recruited across four sites: (1) NSW, Greater Sydney (Greater Capital City Statistical Area, 1GSYD) ${ }^{33}$ (2) Other NSW (eg, Hunter and Illawarra regions), (3) QLD (100 km radius from Brisbane) and (4) WA (600 km radius from Perth). Schools who previously expressed an interest in participating in the trial will be approached initially using contact details provided directly to the research team. In addition, eligible schools across the three states will be approached using publicly available contact details. School principals and health education staff will be sent an invitation outlining the study aims and seeking permission to implement the study. Schools will also be followed up via phone calls and emails from the research staff.

\section{Randomisation}

Following school consent, randomisation, stratifying by state/region and school gender mix (coeducational, predominately male $(>60 \%)$, or predominately female $(>60 \%)$ ) will be conducted. A biostatistician with no role in school recruitment will randomise schools using the Blockrand function in $\mathrm{R}$. The randomisation scheme will be weighted by the total pool of available schools per state/region such that $50 \%$ of the total number of schools recruited to the trial will be recruited from NSW $(25 \%$ from Greater Sydney and 25\% from other regions), while $25 \%$ will be recruited each from WA and QLD. As is the case for school-based interventions of this kind, students, teachers and research assistants will not be blind to intervention allocation. Figure 1 provides an overview of the recruitment and randomisation process.

\section{Informed consent}

Participating schools will be asked to distribute information and consent forms to the parents/guardians via hardcopy or electronically (depending on school preference). Due to differing requirements across ethics committees, some schools will use opt-out (passive) parental consent procedures, while others will require opt-in (active) parental consent. If insufficient opt-in written parent consent is obtained from a school, verbal parental consent will then be sought from the remaining parents by a designated school staff member using contact details available to the school. School staff members who obtain verbal consent will either be reimbursed for their time and effort, or the school will be reimbursed the costs of employing a casual teacher for a day. Students will be provided online participant information statements and required to actively consent prior to the baseline assessment. Consent will also be sought from year 7 teachers at both control and intervention schools to complete online evaluation and fidelity questionnaires. Examples of student, teacher and parent consent forms are included in online supplementary files 1-3.

\section{Patient and public involvement}

Consultation was conducted with adolescents throughout the design phase to inform development of the Health4Life Initiative. This occurred via an online survey $(\mathrm{n}=815$ adolescents) and informal consultation with youth recruited through personal and professional networks. User testing was conducted with students and teachers from two secondary schools to provide feedback about the acceptability and relevance of the intervention, prior to its implementation in schools. Modifications were made to the Health4Life intervention on the basis of this feedback. The programme also underwent expert review to ensure that it reflected the latest scientific evidence.

\section{The Health4Life Initiative}

Schools allocated to the intervention condition will implement the Health4Life intervention in 2019. Health4Life is an eHealth programme that aims to empower adolescents to improve their health and well-being and reduce chronic disease risk by providing simultaneous education about the Big 6 risk factors for chronic disease: physical inactivity, poor diet, sedentary recreational screen time, poor sleep and alcohol and tobacco use. Health4Life adopts a staged model of prevention comprising (1) a universal school-based programme and companion smartphone app delivered to all students regardless of risk, and (2) a selective intervention offering additional web and app content outside of school, delivered students who remain at risk of chronic disease 1 and 2 years after initial intervention delivery (figure 2). This staged prevention approach aims to deter risk factors from emerging in the first place, as well as providing additional support to youth showing indicators of emerging risk behaviours as they progress throughout high school.

\section{The Health4Life school-based programme}

Based on the effective Climate Schools substance use prevention programmes, ${ }^{34-36}$ the first component consists of six, 20 min web-based modules delivered via interactive cartoon storylines about a group of teenagers (figure 3). Modules are ideally delivered once per week for a 


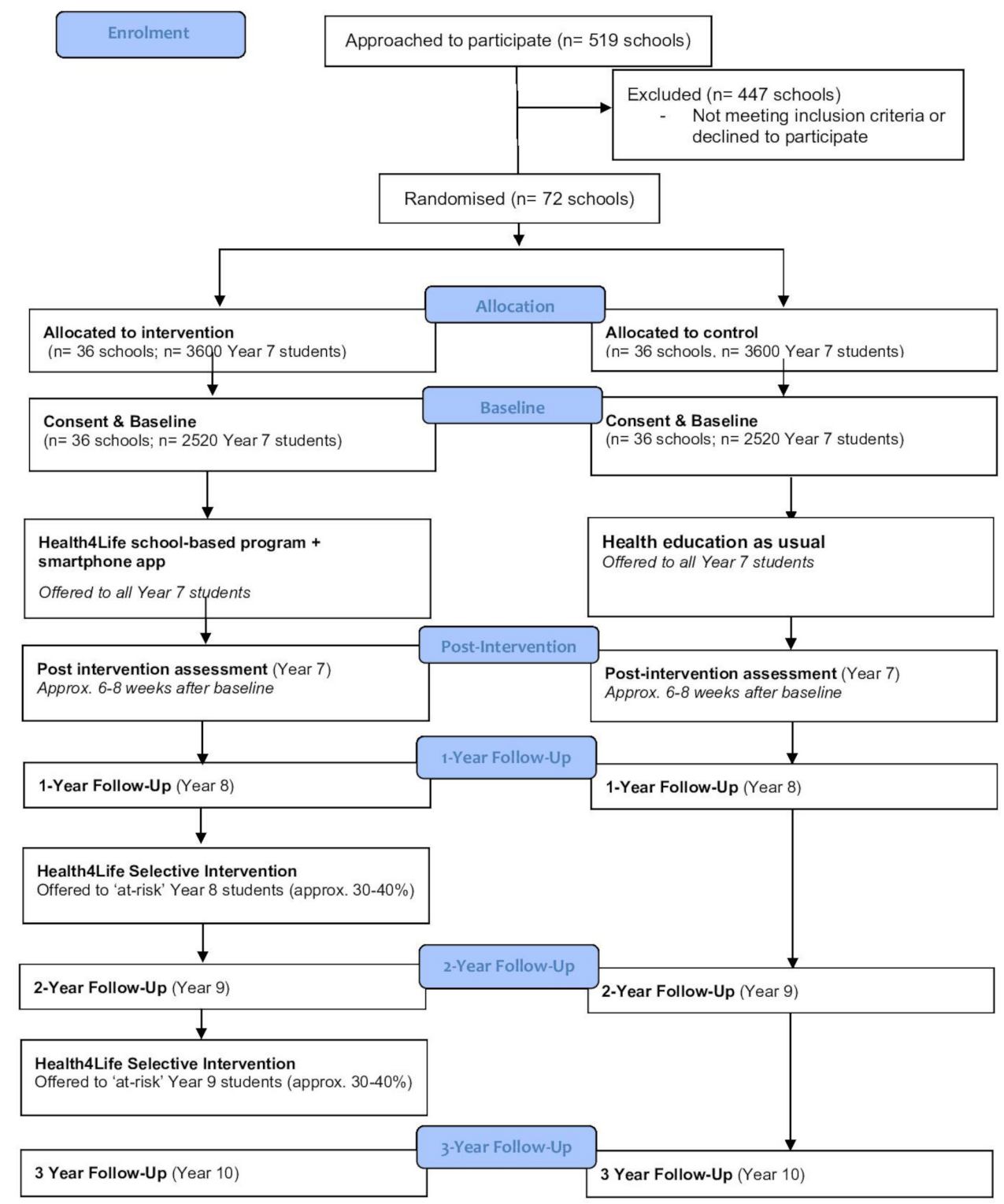

Figure 1 Anticipated recruitment, randomisation and assessment of participants.

period of 6 weeks. Guided by an overarching multiple health behaviour change approach, ${ }^{18}$ the Health4Life programme uses principles of social influence, ${ }^{37}$ social cognitive $^{38}$ and self-determination theories, ${ }^{39}$ and the two-process model of sleep ${ }^{40}{ }^{41}$ to prevent the Big 6 risk behaviours. The key behaviour change techniques woven into the cartoon modules include providing students with evidence-based information about the Big 6 risk factors, improving resistance skills, modifying existing norms, developing life skills (eg, decision-making, problemsolving, coping skills, self-control), self-regulatory skills (eg, goal setting, self-monitoring, decision-making), competence, relatedness and autonomy, and increasing autonomous motivation. Importantly, the cartoons aim to demonstrate the associations and interrelations between multiple health behaviours, for example, how increasing physical activity can improve sleep. Further information about the Health4Life content and development process is published elsewhere. ${ }^{42}$ The modules are completed individually by students and short online quizzes are embedded at the end of each module to test knowledge. Teachers and students are provided with factsheets for each module to reinforce key content and teachers are also provided with a selection of optional activities (eg, online worksheets and games, group discussions, homework tasks) to implement with their students after the cartoon (approximately $20 \mathrm{~min}$ ). An overview of the Health4Life content is provided in table 1 .

Students also receive web-based tailored feedback immediately after completing the online assessments (figure 4). Using a traffic light system, students are provided colour-coded strengths-based feedback about each of the Big 6. The feedback aims to help students to identify behaviours to increase, decrease or maintain. Using this system, 'green' notifications will be provided to students when they are meeting national guidelines for a behaviour ('Going Strong'), 'orange' notifications will be sent to students when they are exhibiting some 


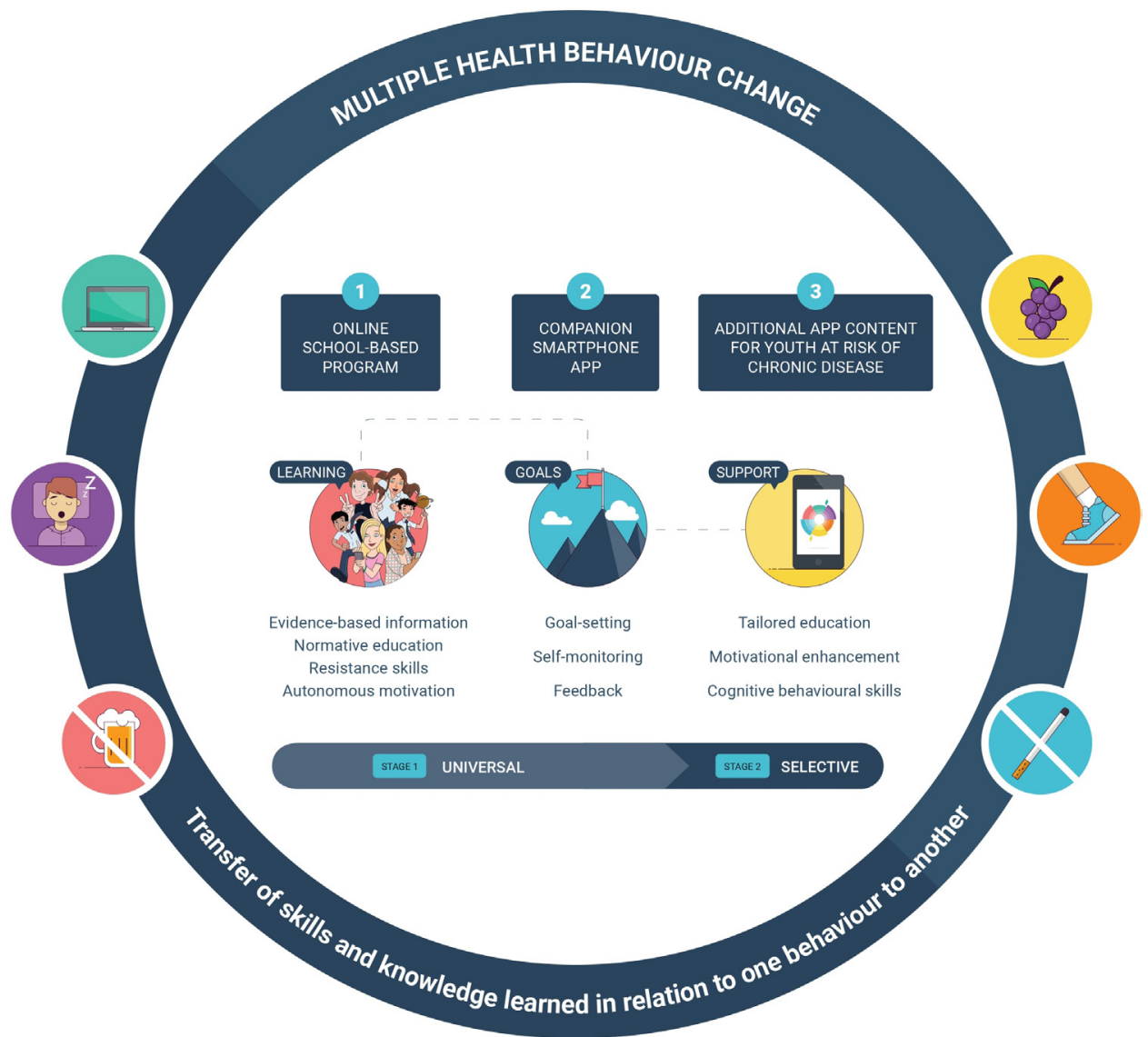

Figure 2 The Health4Life staged model of prevention.

risk or are not yet meeting the guideline, but are close ('Needs some work') and 'red' notifications are provided to students when their behaviour is considered most at risk ('Action required'). All intervention materials will be accessed via the study website (www.health4life.org.au). The programme is aligned with the Australian Health and Physical Education and NSW Personal Development,

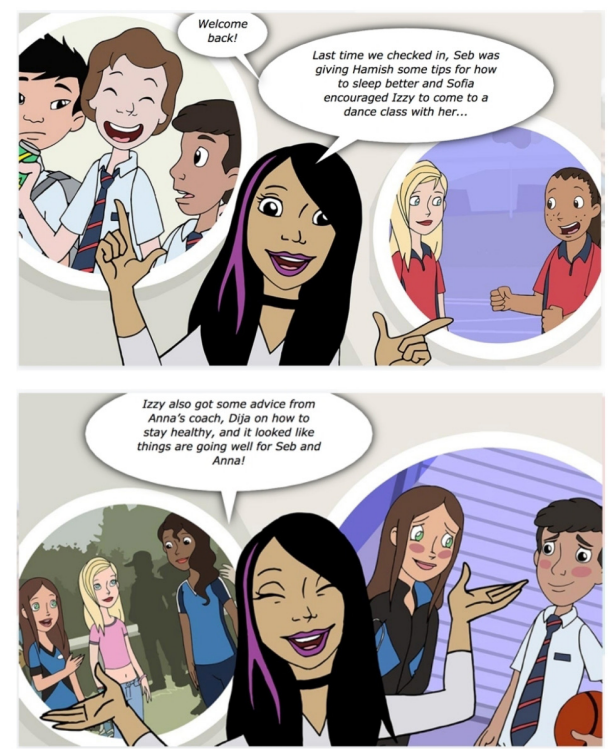

Figure 3 The Health4Life school-based programme.
Health and Physical Education curricula, and is delivered during year 7 health education classes, approximately once per week over 6 weeks. Teachers will be provided with online implementation guidelines and an opportunity to meet with researchers (face-to-face or via video conference) to assist in navigating the website and integrating the programme into their lesson plans; however, no formal teacher training is required.

\section{The Health4Life smartphone app}

To reinforce content taught in the school-based programme and encourage uptake of healthy behaviours, an accompanying smartphone app will be made available to students (see figure 5). All students in the intervention group will be invited to download the Health4Life app and to use the app outside of the classroom. The app is also accessible on tablet devices to encourage ease of access for all students. The app prompts students to enter data to monitor their physical activity, sedentary recreational screen time, dietary intake (servings of fruit/vegetables, SSBs and discretionary food items), abstinence from using alcohol and tobacco, sleep duration and mood, and to view their progress over time. The app also includes goal-setting opportunities and provides motivational quotes and badges/rewards. Students are able to choose how often to use the app; it is anticipated that logging health behaviours would take no more than $10 \mathrm{~min} /$ day. 


Table 1 Overview of Health4Life school-based programme
content

\begin{tabular}{|c|c|}
\hline Module & Key messages \\
\hline 1 & $\begin{array}{l}\text { Guidelines for eating healthily and } \\
\text { benefits of a healthy diet } \\
\text { Tips for increasing water intake } \\
\text { Sleep needs for adolescents and } \\
\text { benefits of sleeping well } \\
\text { Guidelines for recreational screen time } \\
\text { and benefits of limiting screen use }\end{array}$ \\
\hline 2 & $\begin{array}{l}\text { Prevalence and patterns of alcohol and } \\
\text { tobacco use among adolescents } \\
\text { Australian guidelines to reduce health } \\
\text { risks from drinking alcohol } \\
\text { Identifying reasons why teenagers } \\
\text { choose to, or not to, drink alcohol } \\
\text { Benefits of being physically active } \\
\text { - Finding physical activities that you enjoy }\end{array}$ \\
\hline 3 & $\begin{array}{l}\text { Short-term and long-term consequences } \\
\text { of alcohol and tobacco use } \\
\text { Consequences of excessive sedentary } \\
\text { recreational screen time } \\
\text { Strategies for reducing sedentary } \\
\text { recreational screen time } \\
\text { Responsible use of social media }\end{array}$ \\
\hline 4 & $\begin{array}{l}\text { Social, financial and legal consequences } \\
\text { of alcohol and tobacco use } \\
\text { Assertive communication skills and } \\
\text { refusal skills } \\
\text { - Australian guidelines for physical activity } \\
\text { and sedentary behaviour } \\
\text { Tips for setting SMART goals }\end{array}$ \\
\hline 5 & $\begin{aligned} \text { Understanding food labels } \\
\text { Limiting sugar-sweetened beverage } \\
\text { consumption } \\
\text { Improving sleep hygiene } \\
\text { Avoiding too much sleep on weekends } \\
\text { ('social jet lag') }\end{aligned}$ \\
\hline 6 & $\begin{array}{l}\text { Associations and interrelations between } \\
\text { health habits } \\
\text { Relationships between the 'Big 6' and } \\
\text { mental health } \\
\text { Physical, social and emotional benefits } \\
\text { of health and well-being } \\
\text { The Big } 6 \text { and long-term health }\end{array}$ \\
\hline
\end{tabular}

Students will be reminded that they can continue to use the Health4Life app to monitor their behaviour, track their progress and set goals throughout the study period.

The Health4Life selective intervention

Students who are identified as most 'at risk' for two or more health behaviours at the 12-month and/or 24-month follow-up assessment will be invited to access the selective intervention, which consists of additional, tailored content via the Health4Life app or study website. That is, students who fall into the 'red' category ('action required') for two or more risk behaviours using the traffic light system described earlier will be offered access to the additional content when they are in years 8 and 9 , respectively. Based on the WHO four-level approach to school change ${ }^{43}$ we expect that approximately $30 \%$ of students will require this tier of intervention. Table 2 outlines the specific risk thresholds that were developed by the expert advisory group, based on the best available evidence, to target the most at-risk individuals for each behaviour. The sleep threshold was based on the 'Not Recommended' categories for young people aged 5-13 years and 14-17 years, published by the Sleep Health Foundation. ${ }^{44}$ The sedentary recreational screen time threshold was set at $>4$ hours, as research suggests that most young people spend 2-4hours/day engaging in sedentary recreational screen time. ${ }^{45}$ As only $18 \%$ of $12-17$ years old achieve the recommended $60 \mathrm{~min}$ of MVPA each day, ${ }^{4}$ the physical activity threshold was set at 0,1 or 2 days of $60 \mathrm{~min}$ of MVPA in a week. However, given national guidelines state that not drinking is the safest option for this age group, and there is no formal guideline for tobacco use; consumption of a full standard alcoholic drink and any tobacco in the past 6 months was considered at risk. Finally, national guidelines recommend limiting intake of $\mathrm{SSBs},{ }^{46}$ so high intake (5-6 cups/week or 1 or more cups/ day) was considered at risk. Additionally, in recognition of the importance of other key dietary behaviours, a composite risk score based on fruit, vegetable and discretionary food intake could also result in screening into the selective intervention.

The selective intervention will be made accessible to eligible students via an update to the Health4Life app in the second year of the study. Although previous research in socioeconomically disadvantaged schools in Australia found $70 \%$ of student participants reported having access to a smartphone or tablet device, ${ }^{47}$ to increase accessibility, the content will also be available via the Health4Life website. Eligible youth will receive an email and SMS from the research team and 'push notifications' on their smartphones advising them that they can access additional content within the app or the study website to teach them new skills. Designed to be used outside of the classroom setting, the additional content includes tailored education about the Big 6 and uses cognitive behavioural and motivational enhancement techniques to assist students to develop coping strategies and skills to facilitate adoption of health behaviours within their current environment. The selective intervention consists of seven 25 min modules (one for each of the Big 6 and one introductory module). Students will have ongoing access to the selective intervention for the remainder of the study period, with the recommendation that they access it at least once per week until they have completed each module once. 


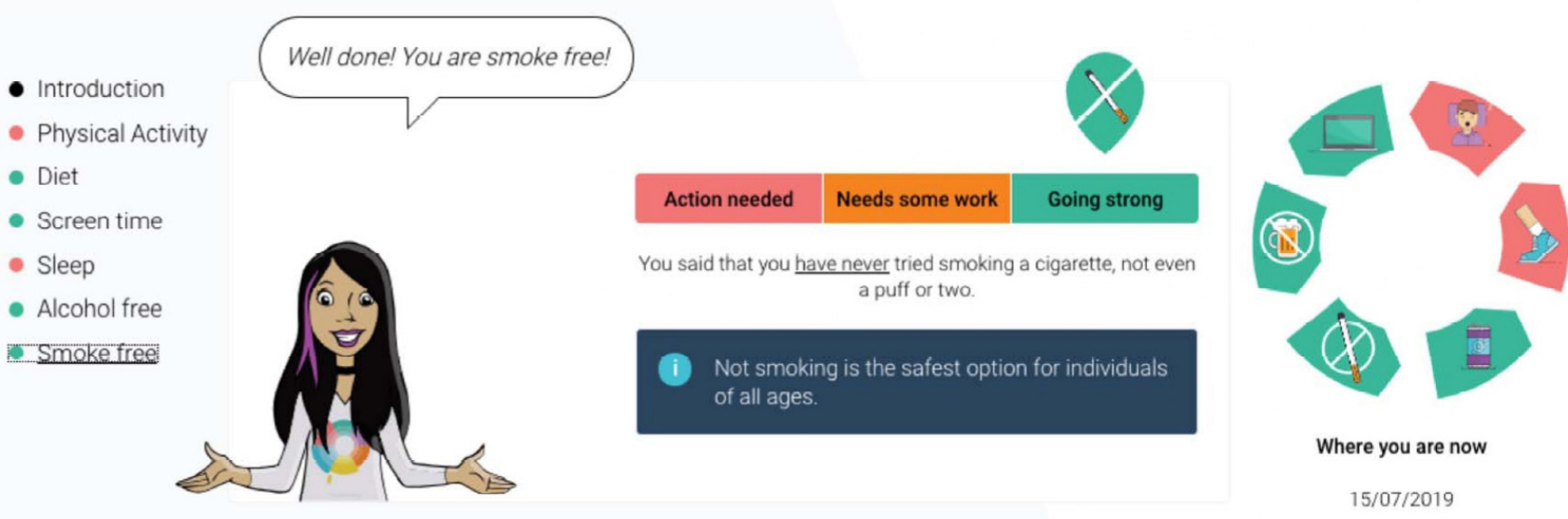

Figure 4 Web-based tailored feedback.

\section{Active control condition}

Control schools will implement health education as usual in 2019, which is mandatory in Australian schools. As such, all control schools will implement curriculum-based health education during the trial (but may vary between traditional teaching and computer/internet-based methods of delivery, depending on each school's regular methods), and this group will serve as an 'active control'. Control schools will be offered complimentary use of the intervention at the end of the study.

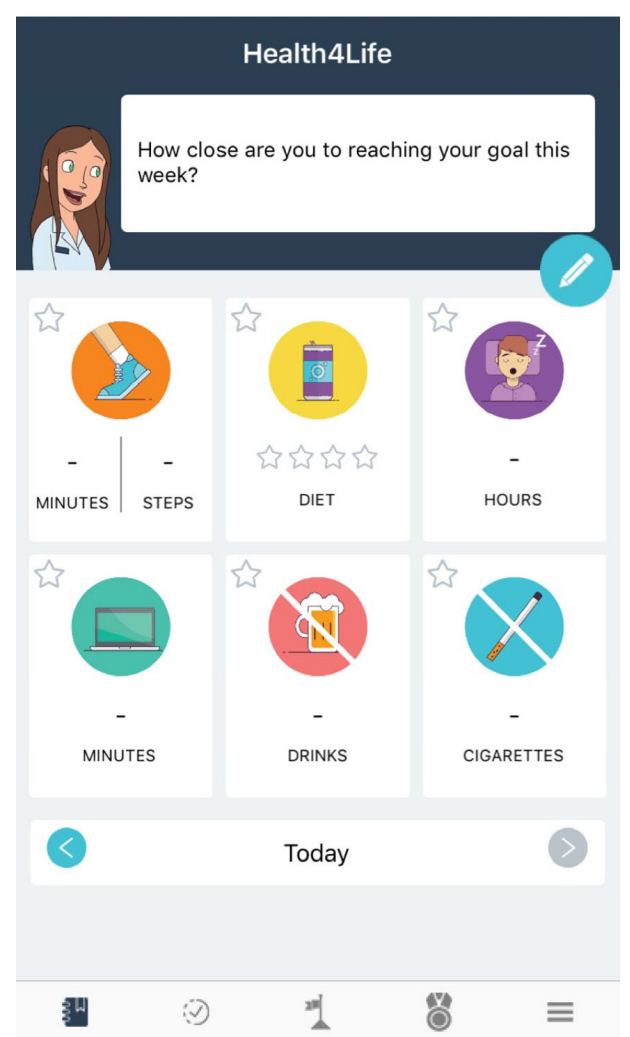

Figure 5 The Health4Life app.

\section{Assessments}

All students will be asked to complete an online selfreport survey in a supervised classroom setting at baseline, immediately post intervention and 12, 24 and 36 months after baseline (table 3 ). Students are required to use their school email address and to create a unique

Table 2 Risk thresholds for the selective intervention.

\begin{tabular}{|c|c|}
\hline Behaviour & Threshold for 'at risk' \\
\hline Alcohol use & $\begin{array}{l}\text { Consumed a full standard alcoholic drink in } \\
\text { the past } 6 \text { months }\end{array}$ \\
\hline Smoking & $\begin{array}{l}\text { Have tried cigarette smoking (even a puff or } \\
\text { two) in the past } 6 \text { months }\end{array}$ \\
\hline $\begin{array}{l}\text { Physical } \\
\text { activity }\end{array}$ & $\begin{array}{l}\text { Engaging in } 60 \text { min of MVPA on } 0,1 \text { or } \\
2 \text { days/week }\end{array}$ \\
\hline $\begin{array}{l}\text { Sedentary } \\
\text { recreational } \\
\text { screen time }\end{array}$ & $\begin{array}{l}>4 \text { hours/day of sedentary recreational } \\
\text { screen time }\end{array}$ \\
\hline \multirow[t]{3}{*}{ Sleep duration } & $\begin{array}{l}<7 \text { or }>12 \text { hours of sleep/night on school } \\
\text { nights if } 6-13 \text { years of age }\end{array}$ \\
\hline & OR \\
\hline & $\begin{array}{l}<7 \text { or }>11 \text { hours of sleep/night on school } \\
\text { nights if } 14-17 \text { years of age }\end{array}$ \\
\hline \multirow[t]{6}{*}{ Diet } & $\begin{array}{l}5-6 \text { cups/week OR } 1 \text { or more cups/day of } \\
\text { sugar-sweetened beverages }\end{array}$ \\
\hline & OR \\
\hline & Two or more of the following: \\
\hline & $-<2$ servings of fruit/day \\
\hline & $-<5$ serves of vegetables/day \\
\hline & $->1$ serve of discretionary food items/day \\
\hline
\end{tabular}

Students are offered the selective intervention when they meet the above criteria for two of more behaviours at the 12-month and/or 24-month follow-up assessment.

MVPA, moderate-to-vigorous physical activity. 
Table 3 Intervention and assessment timeline

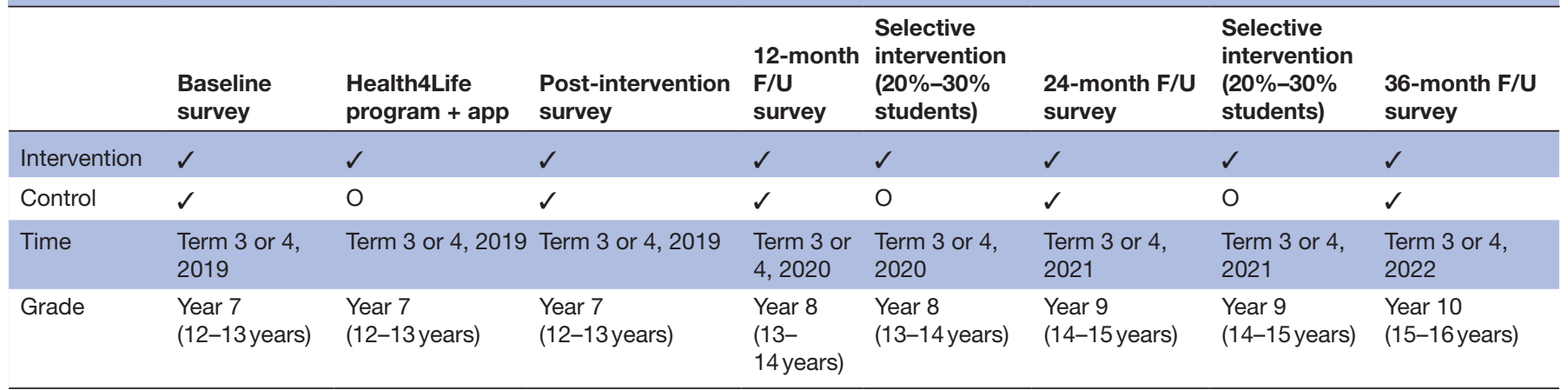

F/U, follow-up.

password to login to complete each survey. Students will be assigned a unique code, which will link data over time while maintaining confidentiality. For follow-up surveys, absent students will be contacted by the research team (using details provided on registration) inviting them to complete the survey at home. Students will be made aware that the contact information they provide will be kept strictly confidential and will be kept secure, separate from their survey responses. After each survey, students will go into the draw to receive a AUD $\$ 100$ gift voucher (two per school/assessment occasion). All teachers will receive a one-off reimbursement of AUD $\$ 100$ for the extra administration required during the first year. These strategies have been successfully used in similar school-based trials with excellent retention rates: $80 \%$ at 24 months, ${ }^{48}$ and $71 \%$ at 36 months ${ }^{36}$ and are deemed acceptable to participating schools.

\section{Measures}

Demographic variables including gender, age, country of birth, language spoken at home and self-reported height, weight. To assess academic performance, students will nominate the range their grades usually fall into $(<49 \%$, $50 \%-59 \%, 60 \%-69 \%, 70 \%-79 \%, 80 \%-89 \%, 90 \%-100 \%)$ and to assess truancy, students will report the number of days they had off school last year without their parents' permission. To measure socioeconomic status, students will complete the Family Affluence Scale III $^{49} 50$ and provide their home postcode, which will be used in addition to each school's Index of Community SocioEducational Advantage score.

\section{Primary outcomes}

Primary outcomes are change over time in self-reported measures of the Big 6 risk factors among the intervention group, relative to change in the control group. The primary endpoint will be the 24-month follow-up.

\section{Sleep duration}

The Modified Sleep Habits Survey ${ }^{51}$ will be used to assess students' sleep patterns. The 6-item scale asks students to report their usual bed time, time attempted sleeping, sleep latency (time taken to fall asleep), time awake after sleep onset, final wake time and time they got out of bed; separately for school nights (Sunday-Thursday) and weekend nights (Friday-Saturday). These items will be combined to produce an average sleep duration (hours and mins), for school nights and weekend nights. Self-reported estimates of bedtime, wake time and sleep duration among adolescents have been shown to be reliable and valid. ${ }^{52} 53$

\section{Sedentary recreational screen time}

Using the International Sedentary Assessment Tool, ${ }^{54}$ students will be asked to recall the past 7 days and report the amount of time (hours and minutes) typically spent in sedentary activity on weekdays and weekend days. Two items will be used to calculate sedentary recreational screen-time-time typically spent sitting, reclining or lying down while (1) watching television or videos during your free time (including watching TV, DVDs, Netflix or online videos) and (2) using an electronic device during your free time (eg, computers, laptops, Xbox, PlayStation, iPads or other tablets, smartphones, YouTube, Facebook or other social media, and the Internet). Respondents are instructed to only report time against their 'main activity' if they were simultaneously watching videos and using an electronic device. Students' average daily screen time will be calculated as a weighted average of weekend and weekday time spent in these two categories of activity combined.

\section{Consumption of SSBS}

SSB consumption will be the primary diet outcome given its strong association with obesity ${ }^{55}$; it is the main source of sugar in the adolescent diet, and prevalence of SSB consumption peaks during adolescence, ${ }^{756}$ whereas consumption of discretionary foods decreases. ${ }^{56}$ Additionally, research suggests that adolescents have the ability to change this behaviour autonomously, ${ }^{57}$ and it is a more equitable diet outcome since it can be switched for tap water at no cost, without altered parental purchasing, unlike fruit, vegetables and discretionary foods. ${ }^{58-60}$ SSB consumption will be assessed using a modified item from the NSW School Physical Activity and Nutrition Survey (SPANS). ${ }^{61}$ Students are provided with a reference image 
that shows the number of metric cups in common sizes of beverages. Students are asked to report how many cups of soft drink, cordials or sports drinks (such as Coke, lemonade, Ribena or Gatorade), excluding energy drinks' they 'usually consume' per week or day. Response options range from 'never/rarely drink', to ' 2 or more cups a day'. Consumption of water, fruit juice and diet drinks are also assessed.

\section{Moderate-to-vigorous physical activity}

A single item taken from the Active Healthy Kids Australia Report Card on Physical Activity ${ }^{4}$ will be used: 'Over the past 7 days, on how many days were you engaged in moderate to vigorous physical activity (activity that increases heart rate and gets you out of breath some of the time) for at least 60 min? Physical activity can be accumulated over the entire day (eg, for example in bouts of $10 \mathrm{~min}$ )'. Students are also asked a similar item with reference to an 'average or typical week'. These items are used to map activity against the Australian 24 hours Movement Guidelines for Children and Young People, ${ }^{62}$ which recommend that young people aged 5-17 years old engage in at least $60 \mathrm{~min}$ of MVPA per day.

\section{Uptake of alcohol use}

Uptake of alcohol use will be assessed using a single item: Have you had a full standard alcoholic drink (see alcohol chart) in the past 6 months?' (Yes/No). A pictorial chart will be supplied to assist participants in identifying what constitutes a standard drink in common portions and types of alcohol. ${ }^{63}$ This question has been used in previous Climate Schools trials ${ }^{3648}$ and is a modified version of an item in the National Drug Strategy Household Survey. ${ }^{64}$

\section{Uptake of tobacco use}

Uptake of tobacco use will be measured using the item 'In the past 6 months, have you tried cigarette smoking, even one or two puffs?' (Yes/No), adapted from the Standard High School Youth Risk Behaviour Survey. ${ }^{65}$

\section{Secondary outcomes}

\section{Mental health symptoms}

Presence of depressive symptoms over the past 7 days will be assessed using the 9-item Patient Health Questionnaire for Adolescents Scale. ${ }^{66}$ Presence of anxiety symptoms over the past 7 days will be assessed via the Patient Reported Outcomes Measurement Information System(PROMIS) Anxiety Paediatric Item Bank. ${ }^{67}$ Internalising and externalising symptoms will be assessed using the Strengths and Difficulties Questionnaire. ${ }^{68}$ Symptoms of psychological distress will be assessed using the Kessler 6 (K6) ${ }^{69}$ Adverse childhood experiences will be assessed using three items adapted from the University of California at Los Angeles Posttraumatic Stress Disorder Reaction Index (UCLA PTSD) Reaction Index, a trauma measure that has been widely used and validated among adolescents aged $7-18 .^{70} 71$
Knowledge about chronic disease risk factors

Knowledge will be measured using a 20-item scale specifically developed to reflect the intended content of Health4Life. Items relating to alcohol and tobacco use were adapted from scales used in previous school-based trials. ${ }^{36} 48$ The scale was derived from a larger pool of items, with user testing among adolescents conducted to condense the scale to 20 items of varying degrees of difficulty. The items assess knowledge of Australian guidelines in relation to physical activity, screen time, sleep, alcohol and dietary intake; prevalence of alcohol and tobacco use among young Australians; and physical and mental health effects of the Big 6 . For each of the 20 statements, students are required to answer 'True', 'False' or 'Don't Know'. Scores are summed to produce a total knowledge score.

\section{Sleep-related outcomes}

The Pediatric Daytime Sleepiness Scale ${ }^{72}$ will be used to assess daytime sleepiness and its relationships to school outcomes, for example, 'How often do you get sleepy or drowsy while doing your homework?'. Five additional items will be used to assess insomnia (difficulty falling asleep), parent-set bedtimes and smartphone use before bed.

\section{Fruit and vegetable consumption}

Intake of fruit and vegetables (serves/per day) will be assessed separately using two questions from the NSW ${ }^{61}$ Students will be presented with images of example servings of fruit and vegetables and asked two how many serves of each they 'usually eat each day'. Response options range from 'I don't eat fruit/vegetables' to 'More than 6 serves per day'.

\section{Discretionary food intake}

To assess intake of discretionary food, students will report the frequency of which they consume six discretionary food items (hot chips, French fries, wedges or fried potatoes; potato crisps or other salty snacks; snack foods, such as sweet and savoury biscuits, cakes, donuts or muesli bars; confectionary; ice cream or ice blocks; and takeaway meals or snacks) from the NSW SPANS. ${ }^{61}$ Response options range from 'never/ rarely drink', to '2 or more times a day'.

\section{Alcohol and tobacco use}

Quantity and frequency of alcohol use will be assessed with the items 'In the past 6 months, how many standard drinks did you have on a typical day when you were drinking alcohol' and 'How often did you have a standard alcoholic drink of any kind in the past 6 months?', respectively. Frequency of binge drinking ( $>5$ drinks on one occasion) will be assessed using 'How often did you have 5 or more standard alcoholic drinks on one occasion in the past 6 months?'. These items are from previous Climate Schools trials. ${ }^{36} 48$ Frequency and quantity of tobacco use will be measured using the items 'During the past 30 days, how many days did you smoke cigarettes?' and 'During 
the past 30 days, on the days you smoked, how many cigarettes did you smoke per day?', adapted from the Standard High School Youth Risk Behavior Survey. ${ }^{65}$

\section{Alcohol-related harms}

To measure alcohol-related harms, students who report drinking alcohol in the past 6 months will complete the Abbreviated Rutgers Alcohol Problem Index. ${ }^{73}$ A 6-item scale adapted from the School Health and Alcohol Harm Reduction Project study ${ }^{74}$ will be used to measure harms caused by others' use of alcohol.

\section{Light physical activity}

To measure light physical activity, a recommendation in the Australia's physical activity guidelines, students will be asked a single item from the Active Australia Survey, ${ }^{75}$ 'In the last week, how many times have you walked continuously, for at least $10 \mathrm{~min}$, for recreation, exercise or to get to or from places?'.

\section{Future intention to adopt health-related behaviours}

Using items from previous school-based trials, ${ }^{36} 48$ participants will be asked to rate how likely they are to use alcohol and tobacco in the future. Responses range from 'very unlikely' to 'very likely'. Students are also asked four items about whether they intend to: 'be physically active on all or most days of the week, ${ }^{76} 77$ 'swap energy drinks, soft drinks, sports drinks or cordial for water on all or most days of the week', reduce my screen time on all or most days of the week' and 'sleep for 9-11 hours per night if aged 5-13 years, or 8-10 hours per night if aged 14-17 years, on all or most days of the week' in the next 3 months. Response options range from 'not at all true of me' to 'very true of me.'

Reliability and validity information for the primary and secondary outcomes along with details of additional outcomes assessed in the student survey are included in online supplementary files 4 and 5 .

\section{Process evaluation}

Process outcomes, such as satisfaction and relevance of the Health4life programme, will also be assessed. Teachers and students in the intervention group will be invited to complete online evaluation questionnaires after completion of Health4Life. Additionally, teachers in the intervention group will be asked to keep a logbook to document their implementation of the programme (ie, timing, activities delivered, technical problems) to assess delivery fidelity. Control teachers will be asked to complete an online survey to give details about health education topics and delivery methods used in 2019. Objective digital metrics of website and smartphone application engagement will also be collected.

\section{Analysis}

Change over time in the multiple risk behaviours associated with the intervention group, relative to change in control group, will be compared using multilevel parallel process growth models. These models account for the relationship across multiple outcomes as well as nesting of repeated measurements within students and students within schools. ${ }^{78}$ As standard practice, the functional form of change across multiple time points will be empirically tested using linear and quadratic growth functions as well as piecewise or discontinuous linear growth functions. Models will be compared using $\chi^{2}$ difference tests of model fit. Conditional models will evaluate whether the intervention has a significant effect (using two-tailed tests of significance) on the change in health behaviours. Models will be estimated using robust maximum likelihood estimation to account for any effects of nonnormality and non-independence of the outcomes. All analyses will be conducted on an intention-to-treat basis, retaining and analysing all students in the groups they were originally allocated. Follow-up exploratory statistical analyses will be carried out to determine whether there is a dose-response relationship between the number of modules students complete and each outcome. Missing data will be explored and if it is reasonable to assume a missing at random structure, will be handled using full information maximum likelihood which yield more efficient, and less biased, parameter estimates than traditional methods of handling missing data.

\section{Data safety, monitoring and quality assurance}

The principal investigator and project coordinator will take responsibility for the management and quality control of study data. Online survey data will be collected via the study website hosted on an external server by a contracted web development company. The external server is based in Australia, and complies with Australian standards in security, ethics and integrity. Survey data will be stored at the University of Sydney. All database files will be password protected, and only direct research personnel will have access to the databases. Study staff who will be accessing data will have appropriate training to maintain confidentiality, data integrity and basic data security measures.

\section{ETHICS AND DISSEMINATION}

This study has been approved by the Human Research Ethics Committees of the University of Sydney (2018/882), the University of Queensland (2019000037) and Curtin University (HRE2019-0083), and all other ethics committees of each participating school, including: the NSW Department of Education (SERAP no. 2019006), the Catholic Education Diocese of Bathurst, the Catholic Schools Office Diocese of Maitland-Newcastle, Edmund Rice Education Australia, the Brisbane Catholic Education Committee (373) and Catholic Education Western Australia (RP2019/07). Results will be disseminated through peer-reviewed publications in medical, health and education journals, at scientific conferences and through webinars and seminars. A summary of the trial results will also be given to all participating schools at the conclusion of the study. Schools will be asked to distribute 
the results to participating students, teachers and parents. Only group aggregate level data will appear in any results or publications.

\section{DISCUSSION}

This will be the first evaluation of an eHealth schoolbased intervention that simultaneously targets six key lifestyle risk factors among adolescents, prior to the onset of chronic disease. The multimodal combination of universal and selective prevention within the one integrated intervention is both novel and at the forefront of prevention in chronic disease. Addressing the Big 6 risk factors during adolescence not only has the potential to improve the health of young Australians in the short term, but also to enhance their capacity to lead healthy lives as adults later in life and reduce their risk of developing chronic disease. By adopting a multiple health behaviour change approach, the Health4Life intervention is a potentially powerful and efficient tool to maximise health outcomes. This evidence-based programme is novel, feasible and scalable to meet the needs of a large number of young people across the country. The online delivery of Health4Life ensures that it has potential to be scaled Australia wide to provide consistent, evidencebased, prevention-focussed health education. To evaluate the feasibility of this approach, the Health4Life study will prioritise recruitment in multiple states, multiple remoteness classifications and multiple school-types Australia wide. However, given the scale of the study, a key limitation includes the reliance on self-report measures and the lack of anthropometric and objective measurement. Nevertheless, this study has the potential to make a substantial public health impact by delaying the growth of the Big 6, thereby reducing the incidence of chronic disease and minimising the associated disability and early mortality.

\footnotetext{
Author affiliations

${ }^{1}$ The Matilda Centre for Research in Mental Health and Substance Use, The University of Sydney, Sydney, New South Wales, Australia

${ }^{2}$ Priority Research Centre for Brain and Mental Health, The University of Newcastle Faculty of Health and Medicine, Callaghan, New South Wales, Australia ${ }^{3}$ Department of Exercise Physiology, University of NSW, Sydney, New South Wales, Australia

${ }^{4}$ School of Education, University of Newcastle, Newcastle, New South Wales, Australia

${ }^{5}$ School of Psychology, University of Queensland, Brisbane, Queensland, Australia ${ }^{6}$ National Drug Research Institute, Curtin University, Perth, Western Australia, Australia

${ }^{7}$ Preventive Medicine, Northwestern University Feinberg School of Medicine, Chicago, Illinois, USA
}

Twitter Maree Teesson @MTeesson, Nicola C Newton @NickieNewton, Frances Kay-Lambkin @ProfFranKayLamb, Cath Chapman @DrCathChapman, Louise Thornton@Louise_Thornton, Tim Slade @DrTimSlade, Lauren A Gardner @ LaurenGardner33, Belinda Parmenter @bjparmenter, Leanne Hides @HidesLeanne, Scarlett Smout @ScarlettSmout and Bridie 0sman @Bridie0sman

Acknowledgements The authors would like to acknowledge Cyanna McGowan, and the schools, students and teachers who participated in the development and testing of the Health4Life intervention.
Collaborators The Health4Life Team: Emma L. Barrett, Louise Mewton, Lexine Stapinski and Louise Birrell.

Contributors $\mathrm{KC}$ and MT led the development of the manuscript and contributed equally to this paper. MT, KC, NN, FK-L, CC, LT, TS, MS, KM, BP, DRL, LH, NM, SA and BS secured funding for the study. MT, KC, NN and LAG are responsible for ethics and governance and overall trial coordination. LAG, BO and SS are responsible for recruitment of schools and data collection in New South Wales. SA and NM are responsible for trial coordination, school recruitment and data collection in Western Australia. LH is responsible for trial coordination, school recruitment and data collection in Queensland. TS and MS are responsible for data analysis and data monitoring. ELB, LM, LS and LB are Associate Investigators and members of the Health4Life team. All authors contributed to developing protocols for the study and reviewed, edited and approved the final version of the paper.

Funding This study is funded by the Paul Ramsay Foundation and the Australian National Health and Medical Research Council (Fellowship to KC, APP1120641; MT, APP1078407; and NN, APP1166377) and via a Centre of Research Excellence in the Prevention and Early Intervention in Mental IIIness and Substance Use (PREMISE; APP11349009).

Disclaimer The funders of the study have no role in study design, data collection, data analysis or data interpretation.

Competing interests None declared.

Patient and public involvement Patients and/or the public were involved in the design, or conduct, or reporting, or dissemination plans of this research. Refer to the Methods section for further details.

Patient consent for publication Not required.

Provenance and peer review Not commissioned; externally peer reviewed.

Open access This is an open access article distributed in accordance with the Creative Commons Attribution Non Commercial (CC BY-NC 4.0) license, which permits others to distribute, remix, adapt, build upon this work non-commercially, and license their derivative works on different terms, provided the original work is properly cited, appropriate credit is given, any changes made indicated, and the use is non-commercial. See: http://creativecommons.org/licenses/by-nc/4.0/.

\section{ORCID iDs}

Maree Teesson http://orcid.org/0000-0002-6744-463X

Katrina E Champion http://orcid.org/0000-0001-8319-9366

Nicola C Newton http://orcid.org/0000-0001-6305-2623

Frances Kay-Lambkin http://orcid.org/0000-0002-4252-5572

Cath Chapman http://orcid.org/0000-0002-2460-6862

Louise Thornton http://orcid.org/0000-0001-7705-833X

Tim Slade http://orcid.org/0000-0002-1725-9188

Matthew Sunderland http://orcid.org/0000-0001-8452-364X

Lauren A Gardner http://orcid.org/0000-0002-8592-6691

Belinda Parmenter http://orcid.org/0000-0001-8013-5658

David R Lubans http://orcid.org/0000-0002-0204-8257

Leanne Hides http://orcid.org/0000-0002-4550-8460

Nyanda McBride http://orcid.org/0000-0003-1714-6631

Bonnie J Spring http://orcid.org/0000-0003-0692-9868

\section{REFERENCES}

1 Ezzati M, Riboli E. Behavioral and dietary risk factors for noncommunicable diseases. N Engl J Med 2013;369:954-64.

2 Telama R, Yang X, Leskinen E, et al. Tracking of physical activity from early childhood through youth into adulthood. Med Sci Sports Exerc 2014;46:955-62.

3 Guerin N, White V. ASSAD 2017 Statistics \& Trends: Australian Secondary Students' Use of Tobacco, Alcohol, Over-the-counter Drugs, and Illicit Substances. Cancer Council Victoria, 2018.

4 Active healthy kids Australia. Physical literacy: do our kids have all the tools? the 2016 report card on physical activity for children and young people. Adelaide, 2016.

5 Morley BSM, Niven P, Wakefield M. National secondary students' diet and activity (NaSSDA) survey, 2012-13. Melbourne, 2014.

6 Evans-Whipp T, Gasser C. Are children and adolescents getting enough sleep? In: Growing up in Australia.The longitudinal study of Australian children (LSAC) annual statistical report 2018. Australian Institute of Family Studies, 2019.

7 Australian Bureau of Statistics. Australian health survey: nutrition first results - food and nutrients, 2011-12. Canberra, 2014. 
8 Cappuccio FP, Taggart FM, Kandala N-B, et al. Meta-Analysis of short sleep duration and obesity in children and adults. Sleep 2008;31:619-26.

9 Hoare $\mathrm{E}$, Milton $\mathrm{K}$, Foster $\mathrm{C}$, et al. The associations between sedentary behaviour and mental health among adolescents: a systematic review. Int J Behav Nutr Phys Act 2016;13:108.

10 Australian Institute of Health and Welfare. Evidence for chronic disease risk factors. Canberra: AlHW, 2016.

11 Ding D, Rogers K, van der Ploeg $\mathrm{H}$, et al. Traditional and emerging lifestyle risk behaviors and all-cause mortality in middle-aged and older adults: evidence from a large population-based Australian cohort. PLoS Med 2015;12:e1001917.

12 Spring B, Moller AC, Coons MJ. Multiple health behaviours: overview and implications. J Public Health 2012;34 Suppl 1:i3-10.

13 de la Haye K, D'Amico EJ, Miles JNV, et al. Covariance among multiple health risk behaviors in adolescents. PLoS One 2014;9:e98141.

14 Barbaresko J, Rienks J, Nöthlings U. Lifestyle indices and cardiovascular disease risk: a meta-analysis. Am J Prev Med 2018:55:555-64.

$15 \mathrm{Li} \mathrm{Y,} \mathrm{Pan} \mathrm{A,} \mathrm{Wang} \mathrm{DD,} \mathrm{et} \mathrm{al.} \mathrm{Impact} \mathrm{of} \mathrm{healthy} \mathrm{lifestyle} \mathrm{factors} \mathrm{on} \mathrm{life}$ Expectancies in the US population. Circulation 2018;138:345-55.

16 Loef $\mathrm{M}$, Walach $\mathrm{H}$. The combined effects of healthy lifestyle behaviors on all cause mortality: a systematic review and metaanalysis. Prev Med 2012;55:163-70.

17 Champion KE, Mather M, Spring B, et al. Clustering of multiple risk behaviors among a sample of 18 -year-old Australians and associations with mental health outcomes: a latent class analysis. Front Public Health 2018;6:135.

18 Prochaska JJ, Spring B, Nigg CR. Multiple health behavior change research: an introduction and overview. Prev Med 2008;46:181-8.

19 Lippke S, Nigg CR, Maddock JE. Health-Promoting and health-risk behaviors: theory-driven analyses of multiple health behavior change in three international samples. Int J Behav Med 2012;19:1-13.

20 Fleig L, Lippke S, Pomp S, et al. Intervention effects of exercise selfregulation on physical exercise and eating fruits and vegetables: a longitudinal study in orthopedic and cardiac rehabilitation. Prev Med 2011;53:182-7.

21 Johnson SS, Paiva AL, Mauriello L, et al. Coaction in multiple behavior change interventions: consistency across multiple studies on weight management and obesity prevention. Health Psychol 2014;33:475-80

22 Geller K, Lippke S, Nigg CR. Future directions of multiple behavior change research. J Behav Med 2017;40:194-202.

23 Hale DR, Fitzgerald-Yau N, Viner RM. A systematic review of effective interventions for reducing multiple health risk behaviors in adolescence. Am J Public Health 2014;104:e19-41.

24 Kipping RR, Howe LD, Jago R, et al. Effect of intervention aimed at increasing physical activity, reducing sedentary behaviour, and increasing fruit and vegetable consumption in children: active for life year 5 (AFLY5) school based cluster randomised controlled trial. BMJ 2014;348:g3256.

25 Busch V, de Leeuw JRJ, de Harder A, et al. Changing multiple adolescent health behaviors through school-based interventions: a review of the literature. J Sch Health 2013;83:514-23.

26 Langford R, Bonell $\mathrm{C}$, Jones $\mathrm{H}$, et al. The world Health organization's health promoting schools framework: a Cochrane systematic review and meta-analysis. BMC Public Health 2015:15:130

27 Sevil J, García-González L, Abós Ángel, et al. Can high schools be an effective setting to promote healthy lifestyles? effects of a multiple behavior change intervention in adolescents. $J$ Adolesc Health 2019;64:478-86.

28 Champion KE, Newton NC, Barrett EL, et al. A systematic review of school-based alcohol and other drug prevention programs facilitated by computers or the Internet. Drug Alcohol Rev 2013;32:115-23.

29 Champion KE, Parmenter B, McGowan C, et al. Effectiveness of school-based eHealth interventions to prevent multiple lifestyle risk behaviours among adolescents: a systematic review and metaanalysis. Lancet Digit Health 2019;1:e206-21.

$30 \mathrm{Heo}$ M, Leon AC. Sample size requirements to detect an intervention by time interaction in longitudinal cluster randomized clinical trials. Stat Med 2009;28:1017-27.

31 Newton NC, Stapinski L, Slade T, et al. Pathways to prevention: protocol for the cap (climate and Preventure) study to evaluate the long-term effectiveness of school-based universal, selective and combined alcohol misuse prevention into early adulthood. BMC Public Health 2018;18:643.

32 Nherera L, Jacklin P. A model to assess the cost-effectiveness of alcohol education developed for NICE public health guidance on personal, social, health and economic (PSHE) education. London:
National Collaborating Centre for Women's and Children's Health, 2009.

33 ASGS. Australian Statistical Geography Standard. Available: https:// www.abs.gov.au/websitedbs/D3310114.nsf/home/Australian+ Statistical+Geography+Standard+(ASGS)

34 Newton NC, Teesson M, Vogl LE, et al. Internet-Based prevention for alcohol and cannabis use: final results of the climate schools course. Addiction 2010;105:749-59.

35 Newton NC, Vogl LE, Teesson M, et al. Climate schools: alcohol module: cross-validation of a school-based prevention programme for alcohol misuse. Aust N Z J Psychiatry 2009;43:201-7.

36 Teesson M, Newton NC, Slade T, et al. Combined universal and selective prevention for adolescent alcohol use: a cluster randomized controlled trial. Psychol Med 2017;47:1761-70.

37 Botvin GJ. Preventing drug abuse in schools: social and competence enhancement approaches targeting individual-level etiologic factors. Addict Behav 2000;25:887-97.

38 Bandura A. Social foundations of thought and action: a social cognitive theory. Englewood Cliffs, N.J: Prentice-Hall, 1986.

39 Ryan RM, Deci EL. Intrinsic and extrinsic motivations: classic definitions and new directions. Contemp Educ Psychol 2000;25:54-67.

40 Borbély AA. A two process model of sleep regulation. Hum Neurobiol 1982;1:195-204.

41 Daan S, Beersma DG, Borbély AA. Timing of human sleep: recovery process gated by a circadian pacemaker. Am J Physiol 1984;246:R161-83.

42 Champion KE, Gardner LA, McGowan C, et al. A web-based intervention to prevent multiple chronic disease risk factors among adolescents: Co-design and user testing of the Health4Life schoolbased program (Preprint). JMIR Form Res 2020.

43 Tully L. Early intervention strategies for children and young people 8 to 14 years: a literature review, 2007. Available: http://www. community.nsw.gov.au/_data/assets/pdf_file/0009/321597/ research_ei_strategies_review.pdf;

44 Sleep Health Foundation. Sleep needs across the lifespan. Australia: NSW, 2015

45 Salmon J, Tremblay MS, Marshall SJ, et al. Health risks, correlates, and interventions to reduce sedentary behavior in young people. Am J Prev Med 2011;41:197-206.

46 National Health and Medical Research Council. Australian dietary guidelines. Canberra: National Health and Medical Research Council, 2013.

47 Lubans DR, Smith JJ, Skinner G, et al. Development and implementation of a smartphone application to promote physical activity and reduce screen-time in adolescent boys. Front Public Health 2014;2:42.

48 Teesson M, Newton NC, Slade T, et al. The climate schools combined study: a cluster randomised controlled trial of a universal Internet-based prevention program for youth substance misuse, depression and anxiety. BMC Psychiatry 2014;14:1-10.

49 Currie C, Molcho M, Boyce W, et al. Researching health inequalities in adolescents: the development of the health behaviour in school-aged children (HBSC) family affluence scale. Soc Sci Med 2008;66:1429-36.

50 Torsheim T, Cavallo F, Levin KA, et al. Psychometric validation of the revised family affluence scale: a latent variable approach. Child Indic Res 2016;9:771-84.

51 Short MA, Gradisar M, Lack LC, et al. Estimating adolescent sleep patterns: parent reports versus adolescent self-report surveys, sleep diaries, and actigraphy. Nat Sci Sleep 2013;5:23-6.

52 Golley RK, Maher CA, Matricciani L, et al. Sleep duration or bedtime? exploring the association between sleep timing behaviour, diet and BMI in children and adolescents. Int $\mathrm{J}$ Obes 2013:37:546-51.

53 Nascimento-Ferreira MV, Collese TS, de Moraes ACF, et al. Validity and reliability of sleep time questionnaires in children and adolescents: a systematic review and meta-analysis. Sleep Med Rev 2016;30:85-96.

54 Prince SA, LeBlanc AG, Colley RC, et al. Measurement of sedentary behaviour in population health surveys: a review and recommendations. PeerJ 2017;5:e4130.

55 Hu FB. Resolved: there is sufficient scientific evidence that decreasing sugar-sweetened beverage consumption will reduce the prevalence of obesity and obesity-related diseases. Obes Rev 2013;14:606-19.

56 Louie JCY, Moshtaghian H, Rangan AM, et al. Intake and sources of added sugars among Australian children and adolescents. Eur J Nutr 2016;55:2347-55.

57 Vézina-Im L-A, Beaulieu D, Bélanger-Gravel A, et al. Efficacy of school-based interventions aimed at decreasing sugar-sweetened 
beverage consumption among adolescents: a systematic review. Public Health Nutr 2017;20:2416-31.

58 Harrison M, Lee A, Findlay M, et al. The increasing cost of healthy food. Aust N Z J Public Health 2010;34:179-86.

59 Palermo C, McCartan J, Kleve S, et al. A longitudinal study of the cost of food in Victoria influenced by geography and nutritional quality. Aust N Z J Public Health 2016;40:270-3.

60 Reicks M, Banna J, Cluskey M, et al. Influence of parenting practices on eating behaviors of early adolescents during independent eating occasions: implications for obesity prevention. Nutrients 2015;7:8783-801.

61 Hardy LL, Mihrshahi S, Drayton BA, et al. NSW schools physical activity and nutrition survey (spans) 2015: full report. Sydney: NSW Department of Health, 2016.

62 The Australian Government Department of Health. Australian 24-hour movement guidelines for children and young people (5 to 17 years): an integration of physical activity, sedentary behaviour, and sleep. Canberra: Commonwealth of Australia, 2019.

63 ADF. What is a standard drink? Available: https://adf.org.au/insights/ what-is-a-standard-drink/

64 Australian Institute of Health and Welfare. National drug strategy household survey 2016: detailed findings. Canberra: AlHW, 2017.

65 Centers for Disease Control and Prevention. Youth risk behavior survey, 2019.

66 Johnson JG, Harris ES, Spitzer RL, et al. The patient health questionnaire for adolescents: validation of an instrument for the assessment of mental disorders among adolescent primary care patients. J Adolesc Health 2002;30:196-204.

67 Irwin DE, Stucky B, Langer MM, et al. An item response analysis of the pediatric PROMIS anxiety and depressive symptoms scales. Qual Life Res 2010;19:595-607.
68 Goodman R. Psychometric properties of the strengths and difficulties questionnaire. J Am Acad Child Adolesc Psychiatry 2001;40:1337-45.

69 Kessler RC, Andrews G, Colpe LJ, et al. Short screening scales to monitor population prevalences and trends in non-specific psychological distress. Psychol Med 2002;32:959-76.

70 Elhai JD, Layne CM, Steinberg AM, et al. Psychometric properties of the UCLA PTSD reaction index. Part II: investigating factor structure findings in a national clinic-referred youth sample. J Trauma Stress 2013;26:10-18

71 Steinberg AM, Brymer MJ, Kim S, et al. Psychometric properties of the UCLA PTSD reaction index: Part I. J Trauma Stress 2013;26:1-9.

72 Drake C, Nickel C, Burduvali E, et al. The pediatric daytime sleepiness scale (PDSS): sleep habits and school outcomes in middle-school children. Sleep 2003:26:455-8.

73 White HR, Labouvie EW. Towards the assessment of adolescent problem drinking. J Stud Alcohol 1989;50:30-7.

74 McBride N, Midford R, Farringdon F, et al. Early results from a schoo alcohol harm minimization study: the school health and alcohol harm reduction project. Addiction 2000;95:1021-42.

75 Australian Institute of Health and Welfare. The active Australia survey: a guide and manual for implementation, analysis and reporting, 2003.

76 Sutherland R, Wiggers J, Campbell E, et al. Physical activity 4 everyone: outcomes of a multi-component schoolbased physical activity intervention for adolescents. Sydney: NSW Ministry of Health, 2017.

77 Okely AD, Lubans DR, Morgan PJ, et al. Promoting physical activity among adolescent girls: the girls in sport group randomized trial. Int J Behav Nutr Phys Act 2017;14:81.

78 Curran PJ. Have multilevel models been structural equation models all along? Multivariate Behav Res 2003;38:529-69. 